\title{
BMJ Open Neoadjuvant therapy or upfront surgery in advanced endometrial cancer: a systematic review protocol
}

\author{
Amy McCarthy, ${ }^{1}$ Katharine Balfour, ${ }^{1}$ Iman El Sayed,${ }^{2}$ Richard Edmondson, ${ }^{1}$ \\ Yee-Loi Louise Wan (1) ${ }^{1}$
}

To cite: McCarthy A, Balfour K, El Sayed I, et al. Neoadjuvant therapy or upfront surgery in advanced endometrial cancer: a systematic review protocol. BMJ Open 2021;11:e054004. doi:10.1136/ bmjopen-2021-054004

- Prepublication history and additional supplemental material for this paper are available online. To view these files, please visit the journal online (http://dx.doi.org/10.1136/ bmjopen-2021-054004).

Received 01 June 2021 Accepted 11 October 2021

Check for updates

(c) Author(s) (or their employer(s)) 2021. Re-use permitted under CC BY-NC. No commercial re-use. See rights and permissions. Published by BMJ.

${ }^{1}$ Gynaecological Oncology, University of Manchester Faculty of Biology, Medicine and Health, Manchester, UK

${ }^{2}$ Biomedical Informatics and Medical Statistics Department Medical Research Institute, Alexandria University Faculty of Medicine, Alexandria, Egypt

Correspondence to

Dr Yee-Loi Louise Wan;

louise.wan@manchester.ac.uk

\section{ABSTRACT}

Introduction There is no consensus on the optimal treatment strategy for people with advanced endometrial cancer. Neoadjuvant therapies such as chemotherapy and radiotherapy have been employed to try to reduce the morbidity of surgery, improve its feasibility and/or improve functional performance in people considered unfit for primary surgery. The objective of this review is to assess whether neoadjuvant chemotherapy or radiotherapy improves health outcomes in people with advanced endometrial cancer when compared with upfront surgery. Methods and analysis This review will consider both randomised and non-randomised studies that compare health outcomes associated with the neoadjuvant therapy and upfront surgery in advanced endometrial cancer. Potential studies for inclusion will be collated from electronic searches of OVID Medline, Embase, international trial registries and conference abstract lists. Data collection and extraction will be performed according to the Preferred Reporting Items for Systematic Reviews and Meta-Analyses guidelines. The methodological quality of the studies will be assessed using the Risk of Bias 2 and Risk of Bias in Non-randomised Studies of Interventions tools. If appropriate, we will perform a meta-analysis and provide summary statistics for each outcome.

Ethics and dissemination Ethics approval was not required for this study. Once complete, we will publish our findings in peer-reviewed publications, via conference presentations and to update relevant practice guidelines.

\section{INTRODUCTION}

Endometrial cancer is the most common gynaecological cancer in high-income countries and its incidence is rapidly rising. ${ }^{1} \mathrm{By}$ 2040 , it is predicted that the global annual incidence of endometrial cancer will rise to nearly 600 000; a rise of over $50 \%$ since 2018 . $^{2}$

Although 5-year survival for women with early-stage endometrial cancer is over $80 \%$, prognosis is much worse for those with advanced disease. Women with distant metastasis at diagnosis (stage 4 disease) have a 5-year survival of $15 \% .^{3}$

Treatment for women with advanced stage disease is poorly defined. This may involve cytoreductive surgery followed by adjuvant

\section{Strengths and limitations of this study}

- Our protocol will incorporate data from randomised and non-randomised studies to evaluate the effectiveness of neoadjuvant therapies in improving survival and treatment-related morbidity in advanced endometrial cancer.

- We will adhere to Preferred Reporting Items for Systematic Reviews and Meta-Analyses and Grading of Recommendations Assessment, Development and Evaluation guidelines to ensure a systematic and rigorous approach to our data synthesis and reporting.

- Our review will inform the design of future studies that investigate the optimal sequence of treatments for patients with advanced endometrial cancer.

- There may be insufficient studies that meet the eligibility criteria to perform a meta-analysis.

- Including non-randomised studies may introduce more bias and reduce the reliability of summary statistics.

chemotherapy, neoadjuvant chemotherapy or radiotherapy with interval debulking surgery or chemotherapy alone.

Neoadjuvant chemotherapy as an alternative to upfront surgery was first studied in women with serous endometrial cancers. ${ }^{4}$ Serous endometrial cancers tend to have similar patterns of intraperitoneal spread and have a similar molecular phenotype as highgrade serous ovarian cancer, ${ }^{5}$ in which neoadjuvant chemotherapy is already an accepted treatment strategy. Neoadjuvant treatment has been suggested to offer a strategy to reduce the morbidity of debulking surgery and improve the feasibility of complete debulking. ${ }^{6}$ It may also potentially improve functional performance in women considered too unfit for extensive surgery. The survival outcomes for women with ovarian cancer who undergo either primary debulking surgery followed by adjuvant therapy or neoadjuvant chemotherapy are broadly equivalent. ${ }^{7}$ Given the biological similarities seen in advanced 
endometrial cancer, in particular serous endometrial cancers, to high-grade serous ovarian cancers, and a paucity of evidence to suggest which strategy might be appropriate in which situation, it is important to examine the clinical evidence for employing either approach.

With the increasing incidence of endometrial cancer and improvements in overall life expectancy, more women are expected to be affected with advanced stage disease. Reducing the morbidity associated with surgery without compromising oncological outcomes is therefore a priority. ${ }^{89}$ This study aims to systematically review the evidence for neoadjuvant chemotherapy and/or radiotherapy in the treatment of women with advanced endometrial cancer. In doing so, we hope to examine the case for undertaking a randomised trial of the optimal sequence of treatment in advanced endometrial by providing an evaluation of its effectiveness and a summary of the limitations of the available studies in this area.

\section{OBJECTIVES}

To assess whether neoadjuvant chemotherapy or radiotherapy prior to surgery improves health outcomes in people with advanced endometrial cancer compared with upfront surgery followed by adjuvant therapy.

\section{METHODS AND ANALYSIS}

\section{Patient and public involvement}

Patients and the public were involved in the generation and the prioritisation of this research question. ${ }^{10}$ Once completed, the results will be disseminated through peerreviewed publications, our social media channels and via our public engagement events.

\section{Criteria for considering studies for this review}

The criteria for considering studies for this review are summarised in table 1.

\section{Types of studies}

We anticipate that there will be very few randomised controlled trials in this area and that other nonrandomised study designs (eg, non-randomised controlled trials, controlled before-and-after studies, historically controlled studies, cohort studies, casecontrol studies, cross-sectional studies and case series) may need to be included to fully evaluate the benefits and harms of neoadjuvant therapy as an initial treatment for advanced endometrial cancer. To minimise bias, we will only include non-randomised studies that include an upfront surgery group as a comparator. Studies which have statistical adjustment for case mix using multivariable analyses will be graded higher quality than those which do not.

\section{Types of participants}

Adults (over the age of 18 years) with advanced stage endometrial cancer (as diagnosed using any recognised International federation of Gynecology and Obstetrics

\begin{tabular}{|c|c|}
\hline Characteristics & Details of what will be considered \\
\hline Population & $\begin{array}{l}\text { People with a new diagnosis of FIGO stage } 3 \text { and } \\
\text { FIGO stage } 4 \text { endometrial cancer }\end{array}$ \\
\hline Intervention & $\begin{array}{l}\text { Neoadjuvant chemotherapy or radiotherapy prior to } \\
\text { surgery }\end{array}$ \\
\hline Comparator & $\begin{array}{l}\text { Primary surgery followed by adjuvant chemotherapy } \\
\text { or radiotherapy }\end{array}$ \\
\hline Outcomes & $\begin{array}{l}\text { Main outcomes of interest } \\
\text { Overall survival } \\
\text { Progression free survival } \\
\text { Secondary outcomes } \\
\text { Completeness of cytoreduction } \\
\text { Patients who complete intended treatment } \\
\text { Adverse events }\end{array}$ \\
\hline Timing & $\begin{array}{l}\text { Overall survival and progression free survival at } 5 \\
\text { years will be compared } \\
\text { Cytoreductive completeness at end of surgery will } \\
\text { be compared } \\
\text { Proportion of patients receiving both treatments } \\
\text { within } 6 \text { months of initiation of treatment } \\
\text { Adverse events up to } 30 \text { days of completing a } \\
\text { treatment will be considered } \\
\text { Long-term side effects of treatments persisting } \\
\text { after first } 6 \text { months after treatment }\end{array}$ \\
\hline Setting & $\begin{array}{l}\text { Prospective and retrospective studies from any } \\
\text { country will be considered }\end{array}$ \\
\hline
\end{tabular}

FIGO, International federation of Gynecology and Obstetrics.

(FIGO) criteria) deemed fit enough to receive surgery in addition to chemotherapy or radiotherapy in any order.

\section{Types of interventions}

Interventions

- Neoadjuvant chemotherapy (single agent or combinations, for example, carboplatin, paclitaxel) with interval surgery.

- Neoadjuvant radiotherapy (external beam or vaginal brachytherapy) followed by surgery \pm further adjuvant therapies.

\section{Comparison}

- Primary debulking surgery followed by chemotherapy.

- Primary debulking surgery followed by radiotherapy.

Types of outcome

Primary outcomes

- Overall survival, defined as time from randomisation to death from any cause.

- Progression free survival, defined as time from randomisation to diagnosis of a recurrence or death.

\section{Secondary outcomes}

- Extent of resection achieved: defined as either complete macroscopic debulking, optimal debulking $(<1 \mathrm{~cm}$ residual) and suboptimal $(>1 \mathrm{~cm}$ residual). Where it is not possible to distinguish between the latter two classes, the categories 'no residual macroscopic disease' and 'residual macroscopic disease' will be used in recognition of the association seen between poorer outcomes and the presence of residual disease in ovarian cancer. ${ }^{11-15}$ 
- Completion of all modalities of treatment planned, that is, neoadjuvant therapy followed by surgery or surgery followed by adjuvant treatment.

- Adverse events: as defined by the Common Terminology Criteria of Adverse Events (V.5.0). ${ }^{16}$ When evaluating this outcome, we will adopt both a confirmatory and exploratory approach to capture both recognised and unanticipated adverse events. The recognised adverse events we will capture are:

- Direct surgical morbidity (eg, visceral injury, intraoperative haemorrhage, need for bowel resection/ anastomosis/stoma, wound complications, haematoma and surgical collections, ileus, vascular injury, nerve injury, return to theatre).

- Surgically related systemic morbidity (eg, venous thromboembolism, chest infection, acute kidney injury, cardiac events).

- Chemotherapy-related toxicity grouped into haematological, gastrointestinal, genitourinary, skin and neurological toxicities.

- Radiotherapy-related toxicity grouped as above and separated into late ( $>6$ months following treatment) and acute toxicities late $(<6$ months following treatment).

- Quality of life measured on any validated scale.

\section{Search methods for identification of studies \\ Electronic searches}

We will search the following electronic databases to identify potential studies:

- Ovid Medline (1990 to date of search).

- Ovid Embase (1990 to date of search).

- ClinicalTrials.gov (1990 to date of search).

- International Clinical Trials Registry Platform (ICTRP) (1990 to date of search).

See online supplemental materials for proposed search strategy.

The platinum-based chemotherapeutic agents used in neoadjuvant chemotherapy for endometrial cancer were not licensed until the $1990 \mathrm{~s}^{17}$ and trials of their use in the adjuvant setting were not published until the mid-2000s. ${ }^{18}$ We will, therefore, only consider studies conducted from 1990 onwards. To reduce language bias, we will include studies published in any language and translate those not written in English.

We will check all included studies for corrections or retractions and perform a further search if there is a greater than 1 year between the original searches and the submission of the completed review.

\section{Searching other resources}

We will handsearch the reference lists of the included articles and reviews of the topic found from the electronic searches, the conference abstracts from the Annual Meeting of the Society of Gynecologic Oncology, International Gynecologic Cancer Society, European Society of Gynecological Oncology and guidelines published by these societies on their own platforms for further potentially eligible studies. We will also search the WHO ICTRP and ClinicalTrails.gov registries for ongoing or unpublished trials. We will contact the lead investigators of relevant studies for further details if ongoing or unpublished trials are found.

\section{Data collection and analysis}

\section{Selection of studies}

The titles and abstracts of all records identified by the electronic searches will be independently screened by two review authors to determine eligibility for inclusion. We will record our decisions on the Abstrackr platform (http://abstrackr.cebm.brown.edu). We will obtain the full text of any studies being considered for inclusion or where there is insufficient information to make a decision about eligibility. Two authors (AM, KB) will independently review all full texts and select those which meet all the inclusion criteria. Disagreements will be resolved by discussion and through involvement of a third review author (Y-LLW).

We will remove duplicates and consolidate all reports pertaining to the same study into a single record. We will present the outcome of our selection process using a flow diagram as recommended by Preferred Reporting Items for Systematic Reviews and Meta-Analyses statement and a 'Characteristics of excluded studies' table.

\section{Data extraction and management}

Two authors will independently extract data from all included trials using a standardised data collection form. The data collection form will be piloted on three study records to check that sufficient data are extracted to allow for data synthesis and risk of bias assessment. Discrepancies between the information extracted will be highlighted and where this is due to disagreement, this will be resolved through discussion with a third review author when necessary. Where clarification is needed regarding data, we will contact the authors where possible.

We will record details regarding the following aspects of each included study:

- Year of publication.

- Study methodology.

- Study population.

- Sample size.

- Patient demographics including staging work-up, FIGO stage, location of disease, histological subtype.

- Inclusion and exclusion criteria.

- Intervention and comparison including dosing and schedules of chemotherapy and radiotherapy, surgical procedure performed and interval at which surgery was considered.

The outcome data will be extracted as follows:

- Time to event data (eg, overall survival and progression free survival): we will extract the $\log \mathrm{HR}$ and its SE. If these are not reported, we will estimate the log HR and its SE using the methods described by Parmar et $a l^{19}$ 
- Dichotomous events (eg, completion of all treatment modalities and adverse events): we will extract the number of patients in each treatment arm who experienced the outcome of interest and the number assessed for this outcome to estimate a risk ratio (RR).

- Continuous outcomes (eg, quality of life measures): we will extract the value at the endpoint and the SD of the outcome of interest and the number of study participants assessed in each treatment arm to estimate the mean difference between arms and its SE.

\section{Assessment of risk of bias in included studies}

Two reviewers will independently assess risk of bias using the Risk of Bias 2 (RoB2) tool for randomised trials and the Risk of Bias in Non-randomised Studies of Interventions (ROBINS-I) tool for non-randomised studies. This assessment will be focused on the main outcomes of the review (ie, overall survival and progression free survival). Where there is disagreement, this will be resolved by discussion and/or involvement of a third reviewer.

For the purposes of assessing randomised trials, we will consider bias in the following domains: bias arising from the randomisation process, bias due to deviations from intended interventions, bias due to missing outcome data, bias in measurement of the outcome and bias in selection of the reported result. Bias due to deviation from intended interventions will be considered according to the effect of assignment to the intervention at baseline (ie, intention to treat). The signalling questions prescribed by the RoB2 tool will be used to reach a risk of bias judgement for each domain of one of three levels: low risk of bias, some concerns, high risk of bias. These results will be used to provide an overall risk of bias judgement.

For the purposes of assessing non-randomised studies, we consider the following as potential confounders: FIGO stage, histological type, performance status, chronological age, access to chemotherapy/radiotherapy/surgery. These factors are recognised prognostic factors which may also determine the choice of initial intervention. As per the ROBINS-I tool, we will assess bias in the following domains: bias due to confounding, bias in the selection of participants in the study, bias in the classification of interventions, bias due to deviations from intended interventions, bias due to missing data, bias in measurement of the outcome, bias in selection of the reported result. We will assign each domain with a risk of bias judgement of one of either low risk of bias, moderate risk of bias, serious risk of bias, critical risk of bias or no information. We will collate these to arrive at an overall risk of bias judgement.

\section{Dealing with missing data}

We will contact the authors of studies with missing data to obtain the relevant information. If this is not available, then we will consider using the presented summary statistics to estimate an effect size as described by Higgins et $a .^{20}$ We will adjust the risk of bias accordingly and conduct sensitivity analyses to investigate the impact of missing values.

\section{Assessment of heterogeneity}

We will assess the clinical and methodological heterogeneity of studies prior to performing any meta-analyses. Heterogeneity will be explored through the prespecified subgroup analysis as described below. Should there be sufficient similarity between the studies to perform a meta-analysis, we will visually inspect the funnel plots and calculate $\mathrm{tau}^{2}$ and $\mathrm{I}^{2}$ values. A random-effects model will be used to try to incorporate heterogeneity which cannot be explained where there are sufficient studies/ participants.

\section{Subgroup analysis and investigation of heterogeneity}

We anticipate that the following may cause significant heterogeneity between studies and will perform subgroup analysis as appropriate. If possible, meta-regression tests will be used to assess the interactions between groups.

- Stage of tumour: stage III/stage IV; rationale being that clinical decision-making may be influenced by severity of disease.

- Type of neoadjuvant therapy: chemotherapy/radiotherapy; rationale being that benefits and harms may differ between treatment modalities and disease distribution may affect patient suitability for each approach.

- Study design: randomised studies/non-randomised studies; rationale being that different study designs may introduce different biases.

\section{Data synthesis}

A meta-analysis of data will only be conducted if the outcomes from individual studies are comparable enough to be mathematically combined and studies are not too heterogeneous. At least three studies will be required for synthesis of data. If a meta-analysis is not appropriate, this will be explained in the review and we will provide a summary of results of included studies either by summarising effect sizes, combining $p$ values or vote counting based on direction of effect as appropriate. $^{21}$

If it is appropriate to conduct a meta-analysis, RevMan will be used. Adjusted summary statistics will be used where possible.

- For time to event data (eg, overall 5-year survival, disease-free survival and disease-free progression), we will pool the $\log \mathrm{HR}$ for each study.

- For dichotomous outcomes (eg, death, adverse events and proportion of patients requiring surgery), we will pool the RR for each study.

- For continuous outcomes (eg, quality of life or surgical completeness), we will estimate the mean difference between treatments arms.

We will use a random-effects model with inverse variance weighting for any meta-analyses performed. ${ }^{22}$ 


\section{Sensitivity analysis}

We will perform sensitivity analyses to assess the robustness of our results. Notably, we will assess the effect of any imputed data, inclusion of studies at high risk of bias and any other issues which arise from the decisions made as part of the review process.

\section{Presentation of findings}

We will present a tabulated summary of our findings which will include the overall certainty of the evidence for each outcome according to the Grading of Recommendations Assessment, Development and Evaluation (GRADE) approach generated using GRADEpro Guideline Development Tool. ${ }^{23}$ We will use the GRADE checklist to facilitate the evaluation of levels of certainty according to the definitions laid out by the GRADE Working Group. ${ }^{24}$

\section{DISCUSSION}

There is no current consensus on the optimal treatment for women with advanced endometrial cancer. ${ }^{25}$ Typically, a combination of surgery, chemotherapy and/or radiotherapy is used. The optimal sequence of these therapies remains controversial but there is evidence to suggest that the strongest prognostic factor for these patients is the extent of cytoreduction achieved. ${ }^{26}$

Neoadjuvant therapy may facilitate achieving complete macroscopic clearance of disease by reducing the morbidity associated with cytoreductive surgery. There are no published systematic reviews that summarise the current evidence for this approach in endometrial cancer. Multiple guidelines and recommendations advocate the consideration of neoadjuvant therapy in patients in whom it is felt that optimal cytoreduction may not be achieved by primary surgery. ${ }^{27} 28$ Further work is required to, not only collate the available evidence, but also to facilitate the design of future studies to evaluate this approach further.

\section{ETHICS AND DISSEMINATION}

Ethics approval was not required for this study. The protocol has been registered prospectively on PROSPERO (CRD42020219461 November 2020). Once complete, we will publish our findings in peer-reviewed publications, via conference presentations, and use it to update relevant practice guidelines.

Contributors Y-LLW and RE devised the research question, approach and rationale. Y-LLW, AM, KB and IES developed the methods to be used for this review. AM prepared the first draft of the manuscript, which was reviewed and revised by Y-LLW, IES and RE. All authors read and approved the final manuscript.

Funding Y-LLW is funded by an NIHR Academic Clinical Lectureship.

Competing interests None declared.

Patient consent for publication Not required.

Provenance and peer review Not commissioned; externally peer reviewed.

Supplemental material This content has been supplied by the author(s). It has not been vetted by BMJ Publishing Group Limited (BMJ) and may not have been peer-reviewed. Any opinions or recommendations discussed are solely those of the author(s) and are not endorsed by BMJ. BMJ disclaims all liability and responsibility arising from any reliance placed on the content. Where the content includes any translated material, BMJ does not warrant the accuracy and reliability of the translations (including but not limited to local regulations, clinical guidelines, terminology, drug names and drug dosages), and is not responsible for any error and/or omissions arising from translation and adaptation or otherwise.

Open access This is an open access article distributed in accordance with the Creative Commons Attribution Non Commercial (CC BY-NC 4.0) license, which permits others to distribute, remix, adapt, build upon this work non-commercially, and license their derivative works on different terms, provided the original work is properly cited, appropriate credit is given, any changes made indicated, and the use is non-commercial. See: http://creativecommons.org/licenses/by-nc/4.0/.

ORCID iD

Yee-Loi Louise Wan http://orcid.org/0000-0003-1441-6050

\section{REFERENCES}

1 Bray F, Ferlay J, Soerjomataram I, et al. Global cancer statistics 2018: GLOBOCAN estimates of incidence and mortality worldwide for 36 cancers in 185 countries. CA Cancer J Clin 2018;68:394-424.

2 Ferlay J, Soerjomataram I, Dikshit R. Cancer incidence and mortality worldwide: sources, methods and major patterns in GLOBOCAN 2012. Int J Cancer 2013;136:E359-86.

3 Office for National Statistics. Cancer survival in England: adult, stage at diagnosis and childhood - patients followed up to 2018, 2019.

4 Despierre E, Moerman P, Vergote I, et al. Is there a role for neoadjuvant chemotherapy in the treatment of stage IV serous endometrial carcinoma? Int J Gynecol Cancer 2006;16 Suppl 1:273-7.

5 Talhouk A, McAlpine JN. New classification of endometrial cancers: the development and potential applications of genomic-based classification in research and clinical care. Gynecol Oncol Res Pract 2016;3:14.

6 Vandenput I, Van Calster B, Capoen A, et al. Neoadjuvant chemotherapy followed by interval debulking surgery in patients with serous endometrial cancer with transperitoneal spread (stage IV): a new preferred treatment? Br J Cancer 2009;101:244-9.

7 Coleridge SL, Bryant A, Lyons TJ, et al. Chemotherapy versus surgery for initial treatment in advanced ovarian epithelial cancer. Cochrane Database Syst Rev 2019;2019.

8 Gyn onc considerations during COVID-19. Available: https://www. sgo.org/resources/gyn-onc-considerations-during-covid-19/ [Accessed 18 Jun 2020].

9 Ramirez PT, Chiva L, Gerda A. COVID-19 global pandemic: options for management of gynecologic cancers. Int J Gynecol Cancer 2020;30:1-3.

10 Wan YL, Beverley-Stevenson R, Carlisle D, et al. Working together to shape the endometrial cancer research agenda: the top ten unanswered research questions. Gynecol Oncol 2016;143:287-93.

11 Bian C, Yao K, Li L, et al. Primary debulking surgery vs. neoadjuvant chemotherapy followed by interval debulking surgery for patients with advanced ovarian cancer. Arch Gynecol Obstet 2016;293:163-8.

12 Vermeulen CKM, Tadesse W, Timmermans M, et al. Only complete tumour resection after neoadjuvant chemotherapy offers benefit over suboptimal debulking in advanced ovarian cancer. Eur J Obstet Gynecol Reprod Biol 2017;219:100-5.

13 Colombo P-E, Mourregot A, Fabbro M, et al. Aggressive surgical strategies in advanced ovarian cancer: a monocentric study of 203 stage IIIC and IV patients. Eur J Surg Oncol 2009;35:135-43.

14 May T, Altman A, McGee J, et al. Examining survival outcomes of 852 women with advanced ovarian cancer: a multi-institutional cohort study. Int J Gynecol Cancer 2018;28:925-31.

15 Manning-Geist BL, Hicks-Courant K, Gockley AA, et al. A novel classification of residual disease after interval debulking surgery for advanced-stage ovarian cancer to better distinguish oncologic outcome. Am J Obstet Gynecol 2019;221:326.e1-326.e7.

16 Common terminology criteria for adverse events (CTCAE) | protocol development | CTEP. Available: https://ctep.cancer.gov/ protocolDevelopment/electronic_applications/ctc.htm [Accessed 18 Jun 2020].

17 Kelland $L$. The resurgence of platinum-based cancer chemotherapy. Nat Rev Cancer 2007;7:573-84.

18 Bestvina CM, Fleming GF. Chemotherapy for endometrial cancer in adjuvant and advanced disease settings. Oncologist 2016;21:1250-9.

19 Parmar MK, Torri V, Stewart L. Extracting summary statistics to perform meta-analyses of the published literature for survival endpoints. Stat Med 1998;17:2815-34. 
20 Higgins J, Li T, Deeks J. Chapter 6: choosing effect measures and computing estimates of effect | Cochrane training. Cochrane Handbook for systematic reviews of interventions, version 6, 2019. Available: www.training.cochrane.org/handbook [Accessed 18 Jun 2020].

21 McKenzie J, Brennan S. Chapter 12: synthesizing and presenting findings using other methods | Cochrane training. Cochrane Handbook for systematic reviews of interventions, version 6.0, 2019. Available: https://training.cochrane.org/handbook/current/chapter-12 [Accessed 18 Jun 2020].

22 DerSimonian R, Laird N. Meta-Analysis in clinical trials. Control Clin Trials 1986;7:177-88.

23 Mustafa RA, Santesso N, Brozek J, et al. The grade approach is reproducible in assessing the quality of evidence of quantitative evidence syntheses. J Clin Epidemiol 2013;66:736-42.
24 Meader N, King K, Llewellyn A, et al. A checklist designed to aid consistency and reproducibility of grade assessments: development and pilot validation. Syst Rev 2014;3:82.

25 Colombo N, Creutzberg C, Amant F, et al. ESMO-ESGO-ESTRO consensus conference on endometrial cancer: diagnosis, treatment and follow-up. Ann Oncol 2016;27:16-41.

26 Barlin JN, Puri I, Bristow RE. Cytoreductive surgery for advanced or recurrent endometrial cancer: a meta-analysis. Gynecol Oncol 2010;118:14-18.

27 et alSundar S, Balega J, Crosbie E. BGCS uterine cancer guidelines: recommendations for practice, 2017. Available: https://www.rcog. org.uk/globalassets/documents/guidelines/clinical-governanceadvice/clinical- [Accessed 18 Jun 2020].

28 Brooks RA, Fleming GF, Lastra RR, et al. Current recommendations and recent progress in endometrial cancer. CA Cancer J Clin 2019;69:258-279. 